\title{
Philosophiques
}

\section{Vincent Citot, dir., Problèmes épistémologiques en histoire de la philosophie, Montréal, Liber, 2017, 398 pages}

\section{Ophélie Desmons}

Volume 45, numéro 1, printemps 2018

URI : https://id.erudit.org/iderudit/1048634ar

DOI : https://doi.org/10.7202/1048634ar

Aller au sommaire du numéro

Éditeur(s)

Société de philosophie du Québec

ISSN

0316-2923 (imprimé)

1492-1391 (numérique)

Découvrir la revue

Citer ce compte rendu

Desmons, O. (2018). Compte rendu de [Vincent Citot, dir., Problèmes

épistémologiques en histoire de la philosophie, Montréal, Liber, 2017, 398 pages].

Philosophiques, 45(1), 321-328. https://doi.org/10.7202/1048634ar d'utilisation que vous pouvez consulter en ligne.

https://apropos.erudit.org/fr/usagers/politique-dutilisation/ 
plutôt, d'en contester l'hégémonie. Se dégagent donc de l'ouvrage deux grandes figures de l'injustice qui, selon la généalogie proposée par C. Spector, ont cohabité chez les philosophes jusqu'à la fin du XvIII siècle. L'Insensé, qui, au nom d'une nature impérieuse, rejette toute forme de justice incarné avec éclat sous la plume de Sade. L'injuste ordinaire, "profiteur » ou "parasite », qui n'a pas le panache de l'Injuste, mais dont le potentiel de nuisance, pour cette raison même, est tout aussi puissant.

Ainsi, l'idée centrale de cet essai semble bien être que la philosophie ne saurait se contenter de confronter cette dernière figure. Confrontation trop facile, puisque le philosophe et le passager clandestin ont la rationalité en partage. Au-delà de la question du mal, c'est bien à une réflexion sur les limites du discours philosophique que cet ouvrage nous invite. Car la confrontation du philosophe à l'Insensé amène nécessairement à se poser la question du destinataire du discours philosophique, ainsi que celle de sa portée. Si les arguments du logos ne sont d'aucune valeur pour celui qui n'en reconnaît pas la primauté, ne doit-on pas reconnaître que tout l'effort du philosophe se réduit à prêcher des convaincus? Quel est, dès lors, l'enjeu de cette confrontation? Pour C. Spector, il semble qu'incarner l'altérité doive permettre de «mesurer l'ampleur des résistances au discours sur la rationalité du juste ${ }^{14}$ » pour les comprendre, mais aussi tenter de les anticiper. Ainsi, si C. Spector travaille la question, classique s'il en est, du mal moral, il nous semble qu'elle engage, dans le même temps, une réflexion nécessaire sur ce que doit être la philosophie politique, dans sa méthode comme dans ses objets.

LUDMILLA LORRAIN Université Paris 1, Panthéon-Sorbonne

\section{Vincent Citot, dir., Problèmes épistémologiques en histoire de la philosophie, Montréal, Liber, 2017, 398 pages.}

Pourquoi faire de l'histoire de la philosophie? Et comment faire de l'histoire de la philosophie? Telles sont les questions fondamentales qui se posent dans le champ de l'épistémologie de l'histoire de la philosophie. Le premier mérite de l'ouvrage dirigé par Vincent Citot est de placer ces questions au centre du débat. L'ouvrage comble ainsi un vide notable. Il y a en effet quelque chose d'étonnant dans le fait que ces questions soient assez rarement abordées dans les premières années du cursus universitaire alors que l'histoire de la philosophie y occupe une place importante. Tandis que ces questions font la plupart du temps l'objet d'un enseignement spécifique chez les historiens et les sociologues, un étudiant en philosophie peut, sans doute 
jusqu'au doctorat, tout ignorer des problèmes et des débats méthodologiques et épistémologiques de l'histoire de la philosophie.

L'ouvrage rassemble plus d'une quinzaine de contributions, organisées en quatre sections thématiques. Les différentes contributions sont marquées par une hétérogénéité de style et de tradition. Certaines relèvent de la tradition française, tandis que d'autres sont de style plus analytique. Les contributions ne convergent pas non plus vers la défense d'une seule et même thèse. Mais plutôt qu'un défaut, il s'agit là d'un autre mérite de cet ouvrage, qui offre un panorama de différentes positions en épistémologie de l'histoire de la philosophie, sans d'ailleurs prétendre à l'exhaustivité.

Je passerai ci-après en revue les trois sections thématiques essentielles de l'ouvrage, la quatrième étant consacrée à des champs plus spécifiques (esthétique, histoire des sciences et pensée politique).

La première section, justement intitulée "philosophie, histoire, sociologie» regroupe des contributions dont le point commun est de s'intéresser, d'une façon ou d'une autre, à la question de scientificité de l'histoire de la philosophie.

François Dosse aborde cette question par le prisme de l'historiographie de ces disciplines que sont l'histoire et la philosophie. Il part du constat d'un fossé entre historiens et philosophes, et se propose d'en rechercher les fondements institutionnels et théoriques. Dosse rappelle d'abord qu'au $\mathrm{XIX}^{\mathrm{e}}$ siècle, lorsque des historiens français comme Gabriel Monod, CharlesVictor Langlois ou Charles Seignobos se sont donné pour objectif de faire de l'histoire une discipline scientifique, ils ont considéré qu'il leur fallait se détourner de la philosophie. Dire ce qui s'était passé supposait que l'on rompe avec toute philosophie de l'histoire, que l'on se déprenne de l'esprit de système et de la tendance à la généralisation. Dosse évoque également le mariage solide entre historiens et géographes, mariage qui s'est conclu dès le $\mathrm{XIX}^{\mathrm{e}}$ siècle, qui s'est poursuivi au XX $\mathrm{XX}^{\mathrm{e}}$ siècle avec l'école des Annales et la géohistoire de Fernand Braudel, et qui a contribué à mettre les philosophes à l'écart. Il estime également que le concept de mentalité, devenu central dans les années soixante, n'a en rien contribué à rapprocher les historiens et les philosophes, dans la mesure où ce concept accordait une prévalence à la psychologie collective plutôt qu'à des pensées émanant d'individus singuliers. L'auteur considère finalement que des reconfigurations récentes semblent plus favorables à un dialogue entre historiens et philosophes. Y participent selon lui le paradigme herméneutique de Paul Ricoeur, l'approche mémorielle de Pierre Nora qui ne vise pas le passé tel qu'il s'est passé mais ses réemplois ou encore l'histoire des intellectuels de Sirinelli qui redonne de l'importance aux individus.

Vincent Citot affronte quant à lui de façon plus conceptuelle la question de savoir à quelle condition l'histoire de la philosophie pourrait devenir une «science rigoureuse » : "pourquoi donc — écrit-il — l'histoire de la philosophie n'est-elle pas, aujourd'hui, une science humaine comme les autres, 
avec ses hypothèses explicatives, ses modèles théoriques, ses tentatives de vérification ou de falsification par les ressources de l'érudition et de la philologie, sa recherche de causes, de lois, de constantes, de tendances, de récurrences, etc. ? Pourquoi les exégètes, les commentateurs et les doxographes tiennent-ils lieu d'historiens ? ${ }^{1} »$ Citot considère ainsi qu'à la différence de l'exégète, qui lit et interprète les textes afin de déterminer ce qu'ont voulu dire les auteurs, l'historien doit expliquer. Il doit dégager les causes, notamment sociales, qui sont à la source des concepts et qui ne sont pas réductibles à la conscience qu'en avaient les auteurs. Citot considère dès lors que l'histoire de la philosophie ne deviendra une science qu'à condition d'assimiler le point de vue de la sociologie.

Les textes de Louis Pinto et de Stanislas Deprez poursuivent cette réflexion, puisqu'ils cherchent à montrer quel pourrait être l'apport d'une approche sociologique de l'histoire de la philosophie. Pinto souligne l'intérêt d'un regard - celui du sociologue — qui ne cherche pas simplement à commenter les textes mais à les comprendre comme des produits historiques engendrés dans des conditions déterminées. En suivant Bourdieu, il propose d'envisager les philosophes comme des joueurs pris au jeu de la philosophie et qui s'efforcent de faire valoir leurs atouts dans un espace social structuré. Il applique notamment cette réflexion à Foucault, dont il estime qu'il «a inventé une position singulière dans le champ philosophique qui consistait à jouer ou à se jouer des frontières disciplinaires sans jamais aller jusqu'à rompre avec sa discipline d'origine [la philosophie $]^{2} »$.

Deprez quant à lui souligne d'abord les défauts de l'approche, largement répandue, qui consiste à considérer les philosophies du passé comme des contemporains et leurs doctrines comme "un vaste stock d'idées et de concepts $^{3}$ » dans lequel on pourrait puiser à l'envie. Cette idée de philosophia perennis ignore simplement que les textes philosophiques sont également des productions historiques liées à une époque donnée. Deprez souhaite développer une méthode capable de rendre compte des facteurs externes, sans qu'elle ne vienne contredire la prétention à la vérité du texte philosophique. Il pense pouvoir y parvenir en combinant les mérites respectifs de l'histoire intellectuelle de Quentin Skinner et de la sociologie critique de Bourdieu.

L'article, et c'est là l'un de ses points forts, décrit de façon synthétique mais précise ces deux approches. De Skinner, il retient d'abord son opposition à l'idée fort répandue selon laquelle ce qui est philosophique dans un

1. Vincent Citot, «L'histoire de la philosophie comme science rigoureuse », dans Vincent Citot, dir., Problèmes épistémologiques en histoire de la philosophie, Montréal, Liber, 20I7, p. 96.

2. Louis Pinto, "Le sociologue face à l'histoire de la philosophie", dans Vincent Citot, dir., Problèmes épistémologiques en histoire de la philosophie, p. I Iо.

3. Stanislas Deprez, "Pour une histoire rhétorique et critique de la philosophie ", dans Vincent Citot, dir., Problèmes épistémologiques en histoire de la philosophie, p. I 22. 
texte, c'est ce qui transcende les circonstances historiques dans lesquelles ce texte est écrit. Au contraire, pour Skinner, ce que l'interprète doit s'efforcer de comprendre pour comprendre le texte, c'est son contexte. Il faut en particulier saisir les débats dans lesquels l'auteur s'inscrit pour comprendre ce qu'il fait en disant ce qu'il dit ${ }^{4}$. De Bourdieu, il retient principalement l'idée selon laquelle les philosophes se positionnent dans un «champ»: ils sont comme des systèmes de coordonnées, toujours situés les uns par rapport aux autres dans un univers social relativement autonome. Deprez rappelle comment Bourdieu a tenté d'appliquer cette conception à la philosophie de Heidegger, s'opposant alors à l'idée selon laquelle l'univers philosophique serait une parfaite abstraction qui échapperait aux logiques sociales. Néanmoins, selon Deprez, qui développe sa proposition dans une courte dernière section, si Bourdieu, avec les concepts de champ et de distinction, a défini une manière scientifique d'étudier l'histoire de la philosophie, il lui manque une méthode. Pourquoi, par exemple, lorsqu'il s'intéresse à Heidegger, s'attarde-t-il tant sur le terme «Fürsorge» plutôt que sur le terme «Dasein»? L'approche de Skinner est susceptible de combler ce défaut méthodologique puisqu'il nous dit ce qu'il faut observer: la façon dont un auteur détourne un concept de son usage courant, dont il se détourne d'un registre pourtant massivement utilisé par ses contemporains, etc.

Finalement, les contributions réunies dans cette section s'attachent aussi bien à pointer les obstacles d'une histoire de la philosophie scientifique qu'à indiquer les pratiques qui permettent de dépasser ces obstacles, pratiques qui peuvent être développées ou transformées. On pourra toutefois souligner qu'un problème important demeure insuffisamment discuté. Si l'histoire de la philosophie se donne pour mission de devenir scientifique, si elle se donne pour mission de ne plus simplement dire ce que dit l'auteur mais d'expliquer pourquoi un auteur a affirmé ce qu'il a affirmé, écrit ce qu'il a écrit et publié ce qu'il a publiés ${ }^{5}$ quelle place reste-t-il pour l'originalité ? Peut-on chercher à expliquer sans réduire à néant les gestes de rupture, qui semblent pourtant caractériser les philosophies dont on se souvient? De ce point de vue, l'histoire intellectuelle notamment développée par Skinner constitue une ressource qui demeure peut-être trop souvent ignorée dans le contexte épistémologique français.

La deuxième partie de l'ouvrage, intitulée «questionnements épistémologiques et philosophiques ", éclaire différentes pratiques de l'histoire de

4. À ce sujet, voir en particulier le passage où Deprez reconstruit la controverse qui a opposé Yves Charles Zarka et Quentin Skinner: "Pour une histoire rhétorique et critique de la philosophie", dans Vincent Citot, dir., Problèmes épistémologiques en histoire de la philosophie, p. I30-І 3 I.

5. Je paraphrase ici les propos de John Dunn, cités par Stéphane Chauvier dans ce volume: Stéphane Chauvier, "Compréhension réelle et compréhension simulée: deux façons de lire les philosophes du passé ", dans Vincent Citot, dir., Problèmes épistémologiques en histoire de la philosophie, p. 242 , note 23. 
la philosophie, explicitant ce faisant les présupposés sur lesquelles elles reposent.

L'intérêt des contributions de Christophe Giolito et Jean-Michel Muglioni est notamment de revenir sur la tradition française d'histoire de la philosophie qui, bien qu'elle ait largement structuré les pratiques scolaires et universitaires, est aujourd'hui très souvent ignorée. Giolito, analysant les pratiques scolaires, propose ainsi une défense de l'éclectisme de Victor Cousin, qui, dit-il, «ne mêle pas ensemble tous les systèmes; car il n'en laisse intact aucun; [...] décompose chacun d'entre en deux parties, l'une fausse, l'autre vraie; $[. .$.$] détruit la première et n'admet que la seconde dans le tra-$ vail de la recomposition ${ }^{6} »$.

Muglioni montre quant à lui ce que la pratique française actuelle de l'histoire de la philosophie en terminales et dans les classes préparatoires doit à Alain qui a été l'instigateur d'une lecture directe des textes, rompant avec les pratiques précédentes, qui privilégiaient les résumés doxographiques et la littérature secondaire. La question que pose l'auteur est dès lors de savoir pourquoi lire directement les textes. La réponse d'Alain, que l'auteur assume également, est très simple: il faut lire directement les textes parce que les grands textes philosophiques font penser. La finalité de la lecture n'est donc pas de savoir ce que dit le texte mais de penser la chose dont il parle.

Muglioni met au jour les présupposés sur lesquels cette conception repose: en premier lieu - et c'est là un point commun avec l'éclectisme de Cousin — l'idée selon laquelle toutes les philosophies contiennent quelque chose de vrai. Deuxièmement, l'idée corrélative qu'il existe " une philosophie éternelle parce que l'humanité ne change pas ${ }^{7}$ ». Les grands textes de l'histoire de la philosophie sont dès lors des lieux privilégiés dans lesquels trouver des éléments de réponse à ces grandes questions transhistoriques que l'humanité se pose. Muglioni assume finalement le caractère anhistorique de cette approche de l'histoire de la philosophie, puisqu'il affirme que cette histoire philosophique de la philosophie est une "histoire sans historicité8 ${ }^{8}$.

On pourra regretter que ces deux contributions ne disent rien des questions de méthode et des problèmes épistémologiques liés à une pratique philosophique de l'histoire de la philosophie. Comment lire les textes pour penser à partir d'eux? Et comment éviter l'écueil de la projection? $\mathrm{Ne}$ devons-nous pas prendre garde au fait que les catégories qui informaient

6. Cf., Victor Cousin, Fragments philosophiques, Genève, Slatkine, I970, p. 75 ; Christophe Giolito, "L'histoire de la philosophie: problèmes et pratique ", dans Vincent Citot, dir., Problèmes épistémologiques en histoire de la philosophie, p. I93, note I7.

7. Jean-Michel Muglioni, «Lire les philosophes », dans Vincent Citot, dir., Problèmes épistémologiques en histoire de la philosophie, p. 207.

8. Ibid., p. 2 I 2. 
«les œuvres de nos plus lointains ancêtres ${ }^{9}$ » ne sont plus celles qui informent notre pensée ? Ne devons-nous pas nous prémunir du risque de ne voir dans ces œuvres que ce que nous y mettons nous-mêmes? Et comment pouvons-nous y parvenir, si ce n'est par un effort de contextualisation?

Les contributions de Frédéric Fruteau de Laclos, Stéphane Chauvier et Pascal Charbonnat se concentrent quant à elles plus précisément sur différentes façons de pratiquer l'histoire de la philosophie.

Stéphane Chauvier place au cœur de son texte une pratique universitaire extrêmement courante, qui consiste à prétendre exposer la philosophie d'un auteur sans la juger. Il propose finalement une critique radicale de cette pratique, qui, sans qu'elle soit nommée, peut être identifiée à l'écriture de monographies. Selon Chauvier, cette pratique ne relève pas l'histoire de la philosophie dont on sait en réalité très bien ce qu'elle est - il cite à titre d'exemples les travaux d'Alain de Libera et de John Dunn. Quelle est dès lors la nature de ce genre qui «consiste en dernière analyse, à s'interdire volontairement d'expliquer historiquement les œuvres philosophiques du passé autant que de les comprendre philosophiquement ${ }^{10}$ ? " Chauvier estime que ce genre est fondamentalement paradoxal dans la mesure où comprendre un énoncé suppose habituellement de le juger. Il considère dès lors que l'auteur de ce type de texte bloque volontairement les conditions du jugement, blocage qui n'est possible que dans le cas d'énoncés qui relèvent de la fiction. Finalement, pratiquer ce genre revient à faire de la pensée d'un auteur une fiction, c'est-à-dire à faire comme si la question de la vérité ne se posait pas à son sujet. À l'appui de cette critique, Chauvier cite, ironiquement mais de manière très convaincante, les propos de Martial Guéroult qui estimait que "[les systèmes philosophiques] valent, comme ils le prétendent, d'une façon exclusive et absolue, ils sont des vérités totales et non partielles, mais chacun dans sa sphère. Or cette absoluité à l'intérieur d'une sphère propre n'est possible que parce qu'il ne s'agit pas pour chacun d'eux de refléter une réalité qui lui est extérieure, mais de constituer chacun une réalité qui lui est propre et intérieure ${ }^{11} »$. Chauvier propose ici une critique radicale de l'un des genres dominants du paysage éditorial, genre qu'il n'estime ni historique ni philosophique.

Frédéric Fruteau de Laclos et Pascal Charbonnat proposent quant à eux d'autres types de pratiques. Fruteau de Laclos prône une pratique distincte de celle de Frédéric Worms, qui se focalise sur les grands «moments » $\mathrm{du} \mathrm{Xx}^{\mathrm{e}}$ siècle, et finalement sur les grandes figures qui sont parvenues à

9. Ibid., p. 208.

10. Stéphane Chauvier, "Compréhension réelle et compréhension simulée: deux façons de lire les philosophes du passé», p. 243.

11. Martial Guéroult, Philosophie de l'histoire de la philosophie, Paris, Aubier, I979, p. 225 ; Stéphane Chauvier, "Compréhension réelle et compréhension simulée: deux façons de lire les philosophes du passé», p. 243 , note 24. 
imposer leurs intérêts philosophiques. Il appelle de ses vœux une pratique qu'il appelle l' " anamnèse » et qui consiste à revenir à des penseurs qui n'ont pas connu de postérité. Fruteau de Laclos souhaite « renouer avec des philosophèmes originaux, oubliés aussitôt qu'ils ont été produits ${ }^{12}$ ". L'enjeu d'une telle pratique est de faire réémerger des possibles qui se sont refermés sitôt ouverts, pour retrouver des pistes qui n'ont pas survécu à l'écriture de l'histoire de la philosophie par les vainqueurs. Fruteau de Laclos applique cette démarche au cas d'Étienne Souriau dont il estime qu'il doit être lu pour lui-même et non comme on le lit le plus souvent (lorsqu'on le lit), à savoir comme un précurseur de Deleuze.

Pascal Charbonnat, qui est historien du matérialisme, propose également un programme de recherche différent. En s'appuyant sur le cas de Diderot qu'il développe tout au long de l'article, il soutient que l'historiographe doit abandonner la tentation de formuler des liens de causalité, parce que de telles affirmations sont toujours incertaines. Ainsi, on ne peut jamais affirmer qu'une expérience scientifique, par exemple l'expérience des animalcules de la farine de blé, a déterminé un énoncé philosophique, par exemple la thèse développée par Diderot selon laquelle la matière est douée de mouvement et de sensibilité. À une telle détermination unilatérale, il faut préférer les concepts de "fonction» et de "réciprocité insaturée ${ }^{13}$ ". Charbonnat recommande aux historiographes de traquer les ressources d'énonciations des conceptions philosophiques en mobilisant les outils de la lexicographie et de s'intéresser à l'environnement plus large et plus diffus dans lequel elles se développent. Il considère que l'historiographie devient scientifique lorsqu'elle est capable de produire un exposé mettant en évidence la solidarité de ces déterminations.

La troisième partie de l'ouvrage est consacrée à l'historiographie des philosophies non occidentales. Le fait d'avoir accordé un espace à ces philosophies souvent ignorées est un autre des mérites de l'ouvrage. De plus, ces pratiques, souvent marquées par une perspective comparatiste, sont remarquables d'un point de vue épistémologique, puisqu'elles font apparaitre en retour les présupposés de l'histoire de la philosophie occidentale, voire les conditions de possibilité de toute histoire de la philosophie.

Ainsi, dans son article intitulé " $\mathrm{La}$ "philosophie» indienne a-t-elle une histoire? », Michel Hulin commence par répertorier les obstacles faisant qu'il n'est en rien évident de répondre positivement à cette question. Premièrement, il est difficile de répondre à cette question parce qu'en Inde aucune

12. Frédéric Fruteau de Laclos, "Comment on écrit l'histoire de la philosophie», dans Vincent Citot, dir., Problèmes épistémologiques en histoire de la philosophie, p. I63.

13. Pascal Charbonnat, "Vers un modèle écologique pour l'historiographie des énoncés philosophiques ", dans Vincent Citot, dir., Problèmes épistémologiques en histoire de la philosophie, p. 252. 
différenciation n'est effectuée entre logos et muthos ${ }^{14}$. Deuxièmement, en raison d'une conception du temps et d'une conception de la vérité qui semblent invalider la possibilité d'une «histoire». La "philosophie» indienne, explique Hulin, se fonde en effet sur une conception cyclique du temps, dans laquelle la vérité est d'emblée donnée au groupe des Brahmanes, vérité dont la transmission est progressivement dégradée avant d'être régulièrement retrouvée. L'idée selon laquelle le savoir est "laborieusement acquis par l'homme » — qui semble en retour constituer l'une des conditions d'une histoire de la philosophie - est totalement absente de ce contexte, dans lequel la notion d'originalité ne peut donc pas non plus avoir de sens.

Nicolas Zufferey insiste quant à lui sur les précautions épistémologiques qui s'imposent à celui qui s'intéresse à la philosophie chinoise, précautions qui, si elles sont ignorées, risquent de conduire l'interprète à conclure hâtivement à l'incohérence des auteurs. En s'appuyant sur sa lecture de Wang Chong, Zufferey souligne lui aussi qu'il faut considérer la conception singulière de la vérité que déploie la pensée chinoise: la vérité n'est pas conçue comme un absolu, indépendant des circonstances; la vérité est à adapter au contexte. Il ajoute qu'il faut mesurer l'importance du statut particulier du penseur chinois, qui est toujours en même temps un conseiller du pouvoir politique. Selon Zufferey, ce n'est qu'en étant sensible à ces éléments de contexte qu'on parvient à retrouver la cohérence du texte et à dépasser les contradictions apparentes.

Finalement, en donnant à voir une diversité d'approches et de pistes, ce volume constitue une contribution essentielle qui alimente et nourrit la réflexion en épistémologie de l'histoire de la philosophie.

OPHÉLIE DESMONS

ESPE de Paris,

Université Paris-Sorbonne

\section{Jacob T. Levy, Rationalism, Pluralism, and Freedom, Oxford, Oxford University Press, 2015.}

Les personnes libres s'associent pour poursuivre les finalités qu'elles partagent. Elles forment des associations telles que des Églises, afin de pratiquer et promouvoir conjointement une religion, ou des universités, pour approfondir et propager les connaissances. Les individus qui partagent une langue ou une origine ethnique se regroupent afin d'exercer leur autonomie politique ou de faire valoir leurs revendications culturelles. Ces groupes sont des associations intermédiaires, ils s'interposent entre l'État et l'individu, jouent

14. Michel Hulin, «La "philosophie" indienne a-t-elle une histoire?», dans Vincent Citot, dir., Problèmes épistémologiques en histoire de la philosophie, p. 298. 